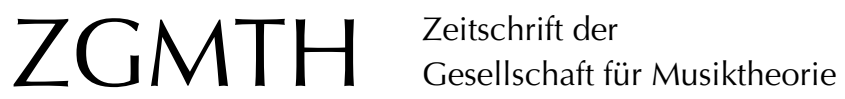

Fladt, Hartmut (2011): Worüber ich nicht sprechen werde. ZGMTH 8/1, 173-176. https://doi.org/10.31751/625

(C) 2011 Hartmut Fladt

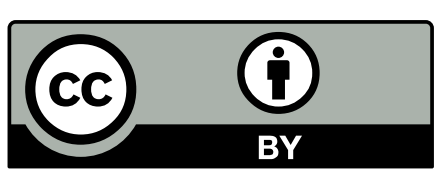

Dieser Text erscheint im Open Access und ist lizenziert unter einer Creative Commons Namensnennung 4.0 International Lizenz.

This is an open access article licensed under a

Creative Commons Attribution 4.0 International License.

veröffentlicht / first published: 14/06/2011

zuletzt geändert / last updated: 21/04/2011 


\title{
Worüber ich nicht sprechen werde
}

\author{
Hartmut Fladt
}

Dieser festliche Titel war fest für meinen Fest-Beitrag im Fest-Akt geplant. Ich halte den Plan wenigstens vorübergehend ein und spreche also jetzt nicht über

- die Neubestimmung der Musiktheorie durch umfassend-kritische Historizität, verbunden mit dem Namen Carl Dahlhaus - von der Propädeutik zur Theorie mit wissenschaftlichem Anspruch zwischen Historie und Systematik;

- ich spreche nicht über uns Achtundsechziger, die wir gern alles musikalisch-Musiktheoretische gesellschaftlich und politisch und politökonomisch zu dechiffrieren versuchten - mit sehr wechselhaftem Erfolg;

- ich spreche nicht über die Wiederentdeckung Schenkers (und erinnere mich an Szenen in der wunderbaren Mühle in Struckum an der Nordsee, wo Michael Polth, Oliver Schwab-Felisch, Ulrich Kaiser und viele andere sich mit mir daran abarbeiteten);

- nicht über die Frustrationen mit den schlechten Abstraktionen der pitch-class-settheory;

- ich verschweige jetzt und hier die von mir so geschätzte erkenntnisträchtige Aufarbeitung von lang-lebigen Improvisations-Modellen und topologischen Traditionen;

- ich spreche nicht über Nelson Goodman: Was haben wir nach der Goodman-Lektüre nicht herumgelabelt, meistens vergeblich, immerhin mit einem jetzt vernünftiger gewordenen Metaphern-Begriff;

- ich spreche nicht über die kritische Aufarbeitung des Hochschul->Fachs` Musiktheorie und seiner Geschichte;

- ich verschweige Neo-Riemann und die Ton-Netze, in denen sich so wenig Beute verfängt;

- $\quad$ und ich spreche nicht über Albert Simon.

Über all das, was ich gerade in der Aufzählung vergessen habe, spreche ich auch nicht mehr. Jetzt sind also einige Seiten dieses ’Journals des Luxus und der Moden der jüngsten Vergangenheit aufgeblättert. Sollte sich dabei früher einmal das Gefühl eingestellt haben: «jetz isse jerettet, die Musiktheorie», dann hat sich das immer sehr schnell als Illusion herausgestellt.

Was ich aber sagen kann: all das hat unserem Fach eine unglaubliche Fülle an Erkenntnis-Gewinn und an Methoden-Vielfalt gebracht. Daran ändert mein demonstrierter satirischer Skeptizismus nichts. Und die GMTH ist die Institution, in der die lebendige, produktive Auseinandersetzung mit all dem ihren zentralen Ort hat. 
Jetzt aber zu meinem heutigen prähistorisch-märchenhaft-mythologischen Anliegen:

\section{Von Höhlenmalereien und Glaubensbekenntnissen}

Als mir einer meiner Wiener Studierenden davon berichtete, wie er in den USA der frühen 90er Jahre (in Miami) in die Kunst eingeweiht wurde, ein Schenker-Diagramm herzustellen, da wurde ich lebhaft an prähistorische Höhlenmalerei erinnert. Eine ihrer wesentlichen Funktionen war es ja, in einem Initiations-Ritus auf Glaubensinhalte einzuschwören - daher die geheimen, unzugänglichen Orte. Das Abbild, die magische Beherrschung des Abgebildeten und die reale Beherrschung der Abbildenden greifen da ineinander. Hinterfragen des Ritus und der Kategorien, so mein Student, wurde ausgeschlossen. Kein kritisches Methoden-Bewusstsein, keine Historizität, keine Alternativen. Musiktheorie als schamanisches Herrschaftsverhältnis. Das gilt selbstverständlich auch für andere Systeme der Musiktheorie, wenn sie so praktiziert werden, und dass es erheblich sinnvollere Umsetzungen von Prinzipien Schenkers gibt, muss nicht besonders hervorgehoben werden.

Andere Ausprägungen der musiktheoretischen Höhlenmalereien sind Resultate von magischem Jagdverhalten: das Bannen der scheuen Beute durch Benennen und Abbilden, durch Umsetzen in eine eigene Symbolik, die Wesenheiten bezeichnen soll. Wer von uns hätte das nicht sozusagen am eigenen Leibe erlebt: Ich beispielsweise bin in zwei Glaubens-Gemeinschaften aufgewachsen - ich war evangelisch-reformiert und funktionstheoretisch. Die Welten ordneten sich wie von selbst, und es wurde reiche Beute gemacht, anerkannt durch mächtige Institutionen. Dann aber kamen die Glaubenszweifel, und sie stellten den Wert dieser Art von Beute heftig in Frage. Auch die magischen Abbildungen der Schemata der sogenannten Formenlehre verloren ihre Faszination. Aber: ich will doch - auch - fasziniert sein, von der Musik, vom Musikverstehen - nur: nicht fremdbestimmt, nicht ideologisch.

Es gibt noch weitere Typen und Deutungsmöglichkeiten von Höhlenmalerei: da sind Symbolsprachen entwickelt, durch die das Jagdbare konkret bezeichnet wird, mit denen auch über Jagdtechniken Auskunft gegeben wird und sogar die Wanderrouten des Jagdbaren festgehalten sind. So nahe mir auch diese Art musiktheoretischer Höhlenmalerei prinzipiell ist - ich weiß selbstverständlich, dass beschwörende Initiations-Riten und magisches Verhalten zur Realität unseres Faches gehören, und ich denke, dass sich auch in meiner Lehre und in meinen Publikationen durchaus Spuren davon finden lassen. Jede musikalische Analyse ist, nüchtern betrachtet, zugleich eine Konstruktion ihres Gegenstandes. Mit dieser Tatsache aber möchte ich kritisch umgehen dürfen, nicht affirmativ oder gar ideologisch.

\section{Vom Rumpelstilzchen-Effekt}

Magisches Verhalten: Da verweise ich immer wieder gern auf das, was ich, ganz märchenhaft, als musiktheoretischen `Rumpelstilzchen-Effekt einem ja bekanntlich aus der Klemme, wenn's darum geht, Stroh, abgedroschenes Zeugs also, in Gold zu verwandeln: Wunschtraum aller Kunst- und Kulturwissenschaft. Aber: 
Rumpelstilzchen stellt Bedingungen. Und: die können fatal sein. Das Erstgeborene dem Dämon ausliefern - vielleicht die Diplomarbeit? Die Seele verkaufen? Sehr fatal, gäbe es nicht ein probates Mittel, vielfach erprobt in Mythen von Naturvölkern, verbreitet als Märchen-Motiv: Das ist die Nennung des Namens als Akt der magischen Bannung.

Hinz, Kunz und Rumpelstilzchen stehen für Wesenheiten: Hinz und Kunz bis heute redensartlich für >jedermann`, das platt-Alltägliche; Rumpelstilzchen dagegen ein Sesamöffne-dich ins Dschinnistan. Die verwirrend chaotische Welt der Erscheinungen scheint "auf den Begriff gebracht", »triffst du nur das Zauberwort«. Und die Macht des ums Feuer tanzenden schwarzen Kobolds ist gebrochen, wenn das Namens-Geheimnis gelöst, der Name benannt ist.

Wenn Kategorien aus Burmeisters Musica poetica oder Bernhards Tractatus compositionis augmentatus für Hypotyposis-, Dissonanz- und Syntax-Figuren benutzt werden, dann ist das nur zu häufig ein Resultat dieses immer wieder begegnenden smagischen Bewusstseins`. Im schlichten Benennen eines Phänomens wird schon sein Erkennen und Bannen vermutet. Dabei liegt doch der kompositorische Witz nicht darin begründet, dass solche Figuren benutzt werden, sondern wie.

Indem Material, Verfahrensweisen und Begrifflichkeiten aller Musik in ihrer immer noch selbstverständlichen Historizität verstanden werden, indem also die Folien rekonstruiert werden, die ja erst die Voraussetzungen für Neuerungen liefern, wird der Verstehens-Prozess in die Wege geleitet. Kulturell verwurzeltes breites geschichtliches VorWissen, immer als Einheit von Er-Innertem, Gefühltem, Gewusstem, Kommuniziertem zu denken, ermöglicht erst jene wunderbar reichen Rezeptionsweisen, die den Großteil der Musik betreffen, die wir kennen und lieben - in allen Genres.

\section{Vom Prokrustes-Prinzip (und Pippi Langstrumpf ist auch dabei)}

Musik in ihren vielen, vielen Ausprägungen und mit ihren ebenso vielen Kontexten ist ein derart komplexes System, dass es kein Wunder ist, wenn nach einheitlichen und konsistenten Erklärungs- und Deutungsmustern gesucht wird. Ach ja, über Luhmann habe ich auch nicht geredet.

Und jetzt kommt ein wissenschaftstheoretischer Lieblingsmythos von mir ins Spiel (nein, nicht Sisyphos, obwohl auch das sehr reizvoll wäre): es ist der liebenswürdige Prokrustes. Wie sagte schon Pippi Langstrumpf so überzeugend: »lch mach' mir die Welt, widde widde wie sie mir gefällt.»

Ich möchte, immer wieder und sehr eindringlich, warnen; warnen vor Systematiken, die der Illusion von Voraussetzungslosigkeit erliegen; sie blenden die Geschichtlichkeiten mit all ihren immer auch semantischen Implikationen schlicht aus. Der mythologische Herr Prokrustes war wissenschaftstheoretisch ein sehr prinzipientreuer, und keine Wirklichkeit konnte ihn schrecken: er fing bekanntlich die vorbeikommenden Reisenden, legte sie auf seine Betten, und wenn sie kleiner waren, wurden sie entsprechend gestreckt, und wenn sie größer waren, schlug er das störend Überhängende ab. So kann sich ein Weltbild ordnen. Peinlicherweise steckt ein Stück Prokrustes in uns allen, auch in mir. Darum: Statt der Tendenz zum folternden Strecken und zum Abhacken im analytischinterpretatorischen Geschäft nachzugeben, rate ich zum selbstverständlichen Offenle- 
gen der eigenen Prämissen, auch zum offenen Bekennen von Unzulänglichkeiten dieser Prämissen.

Analyse-Methoden und Analyse-Systeme, seien sie nun textanalytisch oder höranalytisch intendiert, sind unverzichtbar, und sie sollten dazu da sein, Musik adäquater zu verstehen. Sie haben eine heuristische Funktion. Sich verselbstständigende musiktheoretische Systeme aber tendieren dazu, wie Carl Dahlhaus immer wieder beklagt hat, Musik als Fallbeispiel für die eigene Stichhaltigkeit zu beschlagnahmen. Und was mit dem eigenen System nicht koinzidiert oder ihm gar zuwider läuft und es gefährdet, wird - im besten Falle - ignoriert und im schlechtesten Falle als sentartet bezeichnet.

Die Position unseres so wunderbar reichen Faches Musiktheorie zwischen historischer und systematischer Wissenschaftlichkeit, zwischen analytischer und handwerklicher Kompetenzvermittlung, zwischen kompositorischer/improvisatorischer Kreativität und pädagogischer shandlungsorientierter Reflexion` muss immer wieder als geradezu unglaubliche Chance begriffen werden: `Theorie` eröffnet so ein Potential, das sich in den einzelnen sSparten allein nie ergeben würde. 